\title{
THE PUSH-AND-PULL MECHANISM TO SCAVENGE REDOX-ACTIVE TRANSITION METALS: A NOVEL CONCEPT IN MYOCARDIAL PROTECTION
}

Matthias Karck, MD

Satonori Tanaka, $\mathrm{MD}^{\mathrm{a}}$

Eduard Berenshtein, $\mathrm{MD}, \mathrm{PhD}^{\mathrm{b}}$

Christian Sturm, MD

Axel Haverich, MD

Mordechai Chevion, $\mathrm{PhD}^{\mathrm{b}}$
Objective: Traces of redox-active transition metals such as iron and copper play an important role in free radical formation during postischemic reperfusion of the heart. Two studies were conducted to assess the efficacy of the complexes of desferrioxamine with zinc or gallium to prevent this aspect of reperfusion injury.

Methods: In study I, isolated working rat hearts $(n=96)$ were subjected to 2 hours of hypothermic arrest at $10^{\circ} \mathrm{C}$ induced by use of St Thomas' Hospital cardioplegic solution II supplemented with desferrioxamine, zinc-histidinate, zinc-desferrioxamine, gallium-nitrate, or gallium-desferrioxamine. In study II, isolated nonworking rat hearts $(n=23)$ were subjected to normothermic regional (10 minutes) or global (35 minutes) unprotected ischemia. In this study, the perfusate was supplemented with gallium-desferrioxamine during preischemic and postischemic periods.

Results: In study I, the addition of desferrioxamine, zinc-histidinate, or gallium-nitrate to St Thomas' Hospital solution II improved postischemic aortic flow recovery. When the binary complexes zinc-desferrioxamine or gallium-desferrioxamine were added, however, functional recovery was further enhanced significantly. In study II, high-performance liquid chromatography analyses of tissue from postischemic hearts exposed to unsupplemented perfusate revealed a marked increase of malondialdehydes. In hearts perfused with perfusate supplemented with gallium-desferrioxamine, however, tissue malondialdehyde concentrations were significantly smaller, indicating reduced free radical formation.

Conclusions: The data suggest synergistic protection by the complexes of the iron chelator desferrioxamine with zinc or gallium. The single components neutralize transition metals by 2 different but complementary push-and-pull mechanisms, thereby leading to an inhibition of metal-mediated site-specific free radical formation and improvement of postischemic cardiac function. ( $\mathrm{J}$ Thorac Cardiovasc Surg 2001;121:1169-78)
$\mathrm{R}_{\mathrm{s}}$ eactive oxygen-derived intermediates such as hydrogen peroxides, superoxide anions, and hydroxyl radicals play an important role in postischemic myocardial injury. ${ }^{1}$ Both superoxide anions and hydrogen peroxides are relatively weak oxidants that are neutralized by

From the Department of Thoracic and Cardiovascular Surgery, Hannover Medical School, Hannover, Germany, and the Department of Cellular Biochemistry, ${ }^{\mathrm{b}}$ The Hebrew University, Hadassah Schools of Medicine and Dental Medicine, Jerusalem, Israel.

Copyright (C) 2001 by The American Association for Thoracic Surgery

$0022-5223 / 2001 \$ 35.00+0 \quad \mathbf{1 2 / 1 / 1 1 3 3 2 5}$

doi:10.1067/mtc.2001.113325 endogenous enzymatic or nonenzymatic scavengers such as superoxide dismutase, catalase, or glutathione. However, there are no such scavengers for the highly reactive and cytotoxic hydroxyl radical, which may irreversibly damage cells by lipid peroxidation and alteration of protein structures. ${ }^{2}$ This radical is generated from the superoxide anion and hydrogen peroxide in the presence of trace amounts of transition metal ions of iron or copper via the metal catalyzed Haber-Weiss reaction. ${ }^{3}$ In addition, iron is thought to be necessary for the propagation of lipid peroxidation after its initiation by the hydroxyl radical. ${ }^{4}$ Specific chelators for transition metals such as desferrioxamine (DFO) can tightly bind iron and copper. This effect modulates the redox poten- 


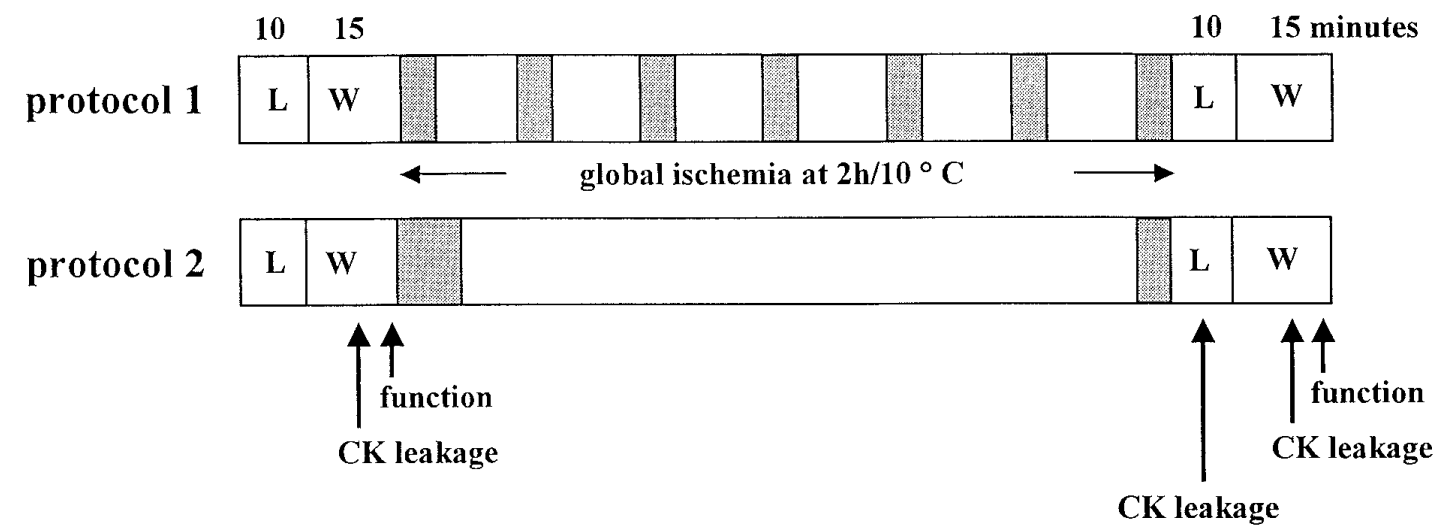

Fig 1. Study I: Experimental time course. $L$, Langendorff perfusion; $W$, working heart perfusion; $C K$, creatine kinase.

tial of these metal ions, and therefore several investigators were able to prove cardioprotective effects of DFO in experimental and clinical studies. ${ }^{5,6}$

On the other hand, redox-inactive zinc ions and also gallium ions compete for the binding sites of redoxactive iron ions, displace them, and divert the site of hydroxyl radical production. ${ }^{7}$ Previous studies have proven that this mechanism is effective for protection of isolated rat hearts. ${ }^{8}$ Likewise, gallium disclosed a protective effect on neonatal rat heart cells subjected to oxidative stress through inhibition of free radical generation. ${ }^{9}$

Because both mechanisms differ in the way they neutralize the presence of iron, there is reason to assume synergistic organ protective effects from DFO in its complexes with zinc or gallium. Recently, they have been identified as protective against postischemic reperfusion injury in the cat retina, ${ }^{10}$ against corneal alkali injury in rabbits, ${ }^{11}$ and against $\mathrm{x}$-ray-induced damage to various tissues. ${ }^{12,13}$ Because available information about the protective capacities of these complexes on the heart is limited, we conducted 2 complementary experimental studies resembling some aspects of clinical cardiac surgery. In study I, isolated working rat hearts were used to assess the effects of zinc-DFO, gallium-DFO, and their constituents alone on the reperfusion injury after hypothermic cardioplegic arrest. In this study, end points included parameters of cardiac function and cellular damage. In study II, nonworking rat hearts subjected to normothermic regional or global ischemia were used to assess the effects of gallium-DFO on tissue concentrations of malondialdehydes, which were measured as an index of lipid peroxidation. In addition, myocardial concentration changes in energy-rich phosphates and their metabolites were assessed.

\section{Materials and methods}

Animals. Male Wistar rats (250-400 g) were used in study I and male Sprague-Dawley rats (250-350 g) in study II. Animals were housed in specific pathogen-free facilities and received standard food and water ad libitum before experiments. All animals received humane care in compliance with the "Principles of Laboratory Animal Care" formulated by the National Society of Medical Research and the "Guide for the Care and Use of Laboratory Animals" prepared by the Institute of Laboratory Animal Resources, National Research Council, and Published by the National Academy Press, revised 1996.

\section{Study I}

Perfusion procedure. The isolated working rat heart model was used in study I. ${ }^{14}$ Rats received sodium heparin (100 IU/100 $\mathrm{g}$ body weight) by intraperitoneal injection. After 20 minutes, the rats were anesthetized by inhalation of diethyl ether. The heart was rapidly excised and placed in ice-cold saline solution. The aorta was then cannulated and Langendorff perfusion was initiated at a pressure of $90 \mathrm{~cm}$ $\mathrm{H}_{2} \mathrm{O}$ with modified Krebs-Henseleit buffer containing (in millimoles per liter) $\mathrm{NaCl}, 118 ; \mathrm{KH}_{2} \mathrm{PO}_{4}, 1.2 ; \mathrm{KCl}, 4.9 ; \mathrm{CaCl}_{2}$, 2.5; $\mathrm{MgSO}_{4}, 1.2 ; \mathrm{NaHCO}_{3}, 25$; and glucose, 11.1. The perfusion fluids were filtered through a $5-\mu \mathrm{m}$ porosity filter to remove any particulate matter. The perfusate was aerated with a mixture of $95 \%$ oxygen and $5 \%$ carbon dioxide $(\mathrm{pH} 7.4)$, and the perfusate temperature was kept at $37^{\circ} \mathrm{C}$. During the time of Langendorff perfusion the left atrium was cannulated for conversion of the preparation to the working heart mode, which was achieved by stopping aortic perfusion and starting left atrial perfusion at a filling pressure of $20 \mathrm{~cm} \mathrm{H}_{2} \mathrm{O}$. Under these conditions the perfusate was ejected spontaneously at a rate of approximately $40 \mathrm{~mL} / \mathrm{min}$ through the aortic cannula 
Table I. Preischemic functional baseline data

\begin{tabular}{|c|c|c|c|c|c|}
\hline Protection & $\begin{array}{c}\text { Aortic flow } \\
\text { (mL/min) }\end{array}$ & $\begin{array}{c}\text { Maximum LVDP } \\
(\text { mm } \mathrm{Hg})\end{array}$ & $\begin{array}{c}d P / d t m a x \\
\left(m m ~ H g / s^{2}\right)\end{array}$ & $\begin{array}{l}\text { Heart rate } \\
\text { (beats/min) }\end{array}$ & $\begin{array}{c}\text { Coronary flow } \\
\text { (mL/min })\end{array}$ \\
\hline \multicolumn{6}{|l|}{ Protocol 1} \\
\hline ST alone & $39.5 \pm 0.8$ & $160 \pm 2$ & $4008 \pm 189$ & $287 \pm 5$ & $20.2 \pm 0.6$ \\
\hline $\mathrm{ST}+\mathrm{DFO}$ & $39.8 \pm 1.0$ & $153 \pm 2$ & $3617 \pm 150$ & $291 \pm 6$ & $19.5 \pm 0.4$ \\
\hline $\mathrm{ST}+\mathrm{Zn}$ & $38.8 \pm 1.2$ & $150 \pm 3$ & $3514 \pm 120$ & $282 \pm 5$ & $19.3 \pm 0.3$ \\
\hline $\mathrm{ST}+\mathrm{Zn}-\mathrm{DFO}$ & $39.8 \pm 0.9$ & $154 \pm 3$ & $3623 \pm 98$ & $293 \pm 3$ & $19.5 \pm 0.4$ \\
\hline $\mathrm{ST}+\mathrm{Ga}$ & $38.5 \pm 0.9$ & $155 \pm 2$ & $3620 \pm 112$ & $274 \pm 4$ & $19.5 \pm 0.3$ \\
\hline $\mathrm{ST}+\mathrm{Ga}-\mathrm{DFO}$ & $39.0 \pm 0.7$ & $153 \pm 2$ & $3562 \pm 101$ & $287 \pm 6$ & $18.5 \pm 0.5$ \\
\hline \multicolumn{6}{|l|}{ Protocol 2} \\
\hline ST alone & $38.8 \pm 1.0$ & $156 \pm 3$ & $3812 \pm 170$ & $288 \pm 7$ & $19.8 \pm 0.9$ \\
\hline $\mathrm{ST}+\mathrm{DFO}$ & $40.8 \pm 0.7$ & $153 \pm 2$ & $3639 \pm 159$ & $283 \pm 4$ & $19.0 \pm 0.5$ \\
\hline $\mathrm{ST}+\mathrm{Zn}$ & $41.3 \pm 0.6$ & $153 \pm 3$ & $3450 \pm 164$ & $293 \pm 3$ & $19.8 \pm 0.2$ \\
\hline $\mathrm{ST}+\mathrm{Zn}-\mathrm{DFO}$ & $38.8 \pm 0.6$ & $154 \pm 2$ & $3564 \pm 166$ & $297 \pm 6$ & $20.0 \pm 0.3$ \\
\hline $\mathrm{ST}+\mathrm{Ga}$ & $38.5 \pm 0.9$ & $149 \pm 2$ & $3588 \pm 96$ & $274 \pm 2$ & $19.0 \pm 0.4$ \\
\hline $\mathrm{ST}+\mathrm{Ga}-\mathrm{DFO}$ & $38.8 \pm 1.0$ & $150 \pm 2$ & $3502 \pm 168$ & $295 \pm 5$ & $18.5 \pm 0.4$ \\
\hline
\end{tabular}

$L V D P$, Left ventricular developed pressure; $d P / d t m a x$, maximum rate of pressure change; $S T$, St Thomas Hospital cardioplegic solution 2; $D F O$, desferrioxamine; $Z n$, zinc, $G a$, gallium.

against a hydrostatic pressure of $90 \mathrm{~cm} \mathrm{H}_{2} \mathrm{O}$. Hearts producing less than $37 \mathrm{~mL} / \mathrm{min}$ aortic flow were excluded from the study. Aortic flow was measured by timed collection of the ejected perfusate in a graduated cylinder. Likewise, coronary flow was measured by timed collection of the coronary effluent. The latter fraction of the perfusate was not recycled. After the measurement of aortic flow, a Millar Mikro-Tip catheter connected to a pressure transducer (Millar Instruments, Inc, Houston, Tex) was inserted into the left ventricle through a rubber-sealed side port of the left atrial cannula for 1 minute of data acquisition. The left ventricular pressure signal and the heart rate were recorded and processed online by an analogdigital converter (Plugsys; Hugo Sachs Electronic, March, Germany). Data processing was performed by an 80386 IBMcompatible personal computer equipped with standard laboratory software (Haemodyn; Hugo Sachs Electronic).

Time course. After cannulation of the aorta, the heart was allowed to stabilize for 10 minutes in the Langendorff perfusion mode (Fig 1). After conversion to the working heart mode, the preparation was perfused for 15 minutes. Contractile function was measured. All hearts were then subjected to 2 hours of global hypothermic ischemia at $10^{\circ} \mathrm{C}$ followed by 10 minutes of normothermic reperfusion in the Langendorff mode. During this time period the duration of ventricular fibrillation until spontaneous conversion to sinus rhythm was recorded. No defibrillator was used. Then, perfusion was converted to the working heart mode and functional recovery was assessed 15 minutes later. Creatine kinase leakage was measured in the coronary effluent collected during the initial 5-minute period of preischemic working heart mode and also during the postischemic Langendorff and working heart perfusion periods. A standard enzymatic test based on spectrophotometric measurement of the amount of nicotinamide adenine dinucleotide phosphate (Granutest 2.5; Merck, Darmstadt, Germany) was used. ${ }^{15}$
Protocol. In hearts protected according to experimental protocol 1, hypothermic cardioplegic arrest was initially induced by 5 minutes of coronary perfusion with St Thomas' Hospital cardioplegic solution II (ST) at $10^{\circ} \mathrm{C}$ via a sidearm of the aortic cannula. The content of ST is as follows (in millimoles per liter): sodium, 120.0; potassium, 16.0; calcium, 1.2; magnesium, 16.0; chloride, 160.4; bicarbonate, 10.0. During ischemia, hearts were subjected to 6 cycles of 15 minutes of no flow followed by 5 minutes of hypothermic perfusion with the same perfusate as used for induction of ischemia. Depending on the experimental group, 8 experiments per group were performed with ST alone or supplemented with DFO $(50 \mu \mathrm{mol} / \mathrm{L})$, zinc-histidinate $(30 \mu \mathrm{mol} / \mathrm{L})$, zinc-DFO $(5 \mu \mathrm{mol} / \mathrm{L})$, gallium-nitrate $(100 \mu \mathrm{mol} / \mathrm{L})$, or gallium-DFO $(5 \mu \mathrm{mol} / \mathrm{L})$. Protocol 2 differed from protocol 1 in that the initial coronary perfusion time for induction of cardiac arrest was extended from 5 to 10 minutes. Unlike in protocol 1, there were no reinfusions during ischemia except for 5 minutes before onset of normothermic reperfusion, when cardioplegic solution was readministered. Depending on the experimental group, supplements to the cardioplegic solution were the same as with protocol 1.

\section{Study II}

Perfusion procedure. The isolated nonworking Langendorff model was used in study II. Rats were injected with sodium heparin (500 units intraperitoneally) 30 minutes before anesthesia with sodium pentobarbital $(60 \mathrm{mg} / \mathrm{kg}$ intraperitoneally). The heart was rapidly excised and placed in ice-cold saline solution. After cannulation of the aorta, hearts were perfused in the Langendorff mode at a pressure of $85 \mathrm{~cm} \mathrm{H}_{2} \mathrm{O}$ with modified Krebs-Henseleit buffer containing (in millimoles per liter) $\mathrm{NaCl}$, $118 ; \mathrm{KCl}, 5.8 ; \mathrm{CaCl}_{2}, 2.5 ; \mathrm{MgSO}_{4}, 1.2 ; \mathrm{NaHCO}_{3}, 25$; and glucose, 11.1. Perfusion fluids were prepared on the day of the experiment and filtered before use. The perfusate was aerated 


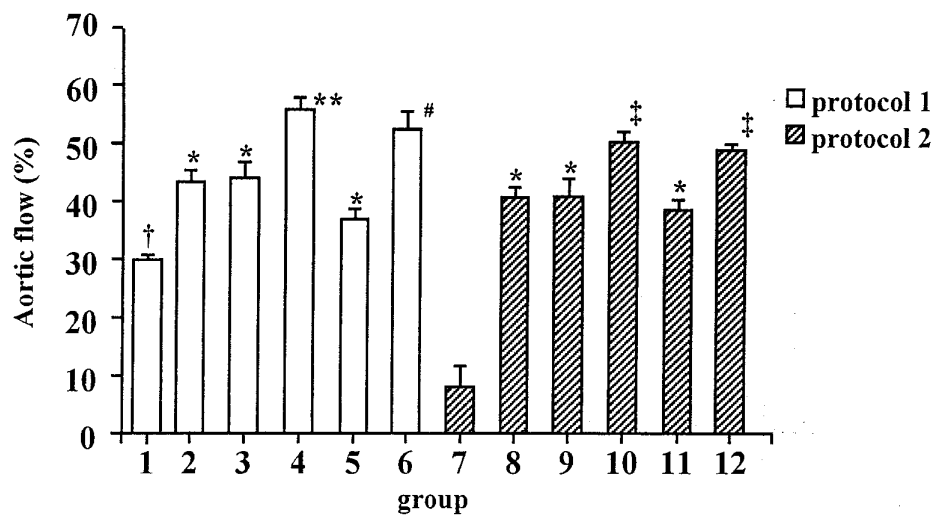

Fig 2. Recovery rates of aortic flow in study I (in percent of preischemic value). 1 and 7, ST alone; 2 and 8 , ST + DFO; 3 and 9, ST + zinc; 4 and 10, ST + zinc-DFO; 5 and 11, ST + gallium; 6 and 12, ST + gallium-DFO. Protocol 1, Intermittent cardioplegia; protocol 2, single-shot cardioplegia. $\dagger P<.05$ versus ST-alone group (protocol 2). $* P<.05$ versus corresponding ST-alone group; $* * P<.05$ versus corresponding groups $\mathrm{ST}+$ zinc, $\mathrm{ST}+$ gallium, $\mathrm{ST}+\mathrm{DFO}$, and $\mathrm{ST}$ alone; $\# P<.05$ versus corresponding groups $\mathrm{ST}+$ gallium, $\mathrm{ST}+\mathrm{DFO}$, and $\mathrm{ST}$ alone; $\ddagger P<.05$ versus corresponding groups ST $+\mathrm{DFO}$ and $\mathrm{ST}$ alone.

with a mixture of $95 \%$ oxygen and $5 \%$ carbon dioxide ( $\mathrm{pH} 7.4)$, and the perfusate temperature was kept at $37^{\circ} \mathrm{C}$. After functional stabilization during 20 minutes of perfusion, hearts were subjected either to 10 minutes of regional ischemia by placing an occlusive snare around the left anterior descending artery (groups 2 and 3) or to 35 minutes of no-flow global normothermic ischemia (groups 4 and 5). Then, hearts were reperfused for 10 minutes (groups 2 and 3) and 20 minutes (groups 4 and 5) before they were freeze-clamped with Wollenberger tongs precooled in liquid nitrogen. The perfusate of hearts in groups 3 and 5 was supplemented with gallium-DFO complex $(5 \mu \mathrm{mol} / \mathrm{L})$ throughout the experiment, whereas hearts in groups 2 and 4 were perfused with unsupplemented buffer. Another 4 hearts were freeze-clamped after the stabilization period (group 1). They served as nonischemic baseline controls.

High-performance liquid chromatography measurements. Malondialdehyde, ascorbic acid, adenosine triphosphate, diphosphate, and monophosphate (ATP, ADP, AMP), and other nucleotides and nucleosides in heart tissue were determined by high-performance liquid chromatography (HPLC) from perchloric acid extracts prepared under liquid nitrogen. The HPLC system consisted of a Varian 5000 liquid chromatograph (Varian Medical Systems, Palo Alto, Calif) equipped with a Rheodyne 7125 sample injector $(20-\mu \mathrm{L}$ loop) (Rheodyne, Rohnert Park, Calif) connected to a linear UVIS 200 detector (UVIS, Reno, Nev). The column used for separation was a 25 $\mathrm{cm} \times 4 \mathrm{~mm}$ supersphere $100 \mathrm{RP}-18,4 \mu \mathrm{m}$ (E. Merck, Darmstadt, Germany) provided with a guard column packed with the same matrix as the separative column. The signals from the detector were acquired on a PC Microsoft MS-DOS version 5.0 data system (Microsoft Corporation, Redmond, Wash) and subsequently processed. The mobile phase consisted of 2 eluents: $10 \mathrm{mmol} / \mathrm{L}$ tetrabutylammonium hydroxide, 10 $\mathrm{mmol} / \mathrm{L} \mathrm{KH}_{2} \mathrm{PO}_{4}, 1 \%$ methanol, $\mathrm{pH} 7.0$ (buffer A) and 2.8 $\mathrm{mmol} / \mathrm{L}$ tetrabutylammonium hydroxide, $100 \mathrm{mmol} / \mathrm{L}$ $\mathrm{KH}_{2} \mathrm{PO}_{4}, 30 \%$ methanol, pH 5.5 (buffer B).
The gradient for chromatographic separation was as follows: 26 minutes of $100 \%$ of buffer A, 2 minutes at up to $40 \%$ of buffer B, 11 minutes at up to $44 \%$ of buffer B, and 10 minutes at up to $100 \%$ of buffer B that is held for an additional 5 minutes. The initial conditions were restored after 18 minutes of washing with buffer A. The flow rate was $0.3 \mathrm{~mL} / \mathrm{min}$, column temperature was $18^{\circ} \mathrm{C}$, and the detection was performed at $266 \mathrm{~nm}$, that is, the maximum of adsorption of malondialdehyde and other compounds. ${ }^{16,17}$

Expression of results. All results are expressed as mean \pm SEM. Creatine kinase leakage, measured during the preischemic control perfusion period and during the first 5 minutes of both Langendorff reperfusion and working heart reperfusion, is expressed as international units per 5 minutes per heart. Preischemic values of cardiac function, including aortic flow, maximum left ventricular developed pressure, maximum rate of pressure change ( $\mathrm{dP} / \mathrm{dtmax})$, heart rate, and coronary flow, are expressed in terms of the absolute values, and indices of postischemic cardiac function are expressed as a percentage of their individual preischemic values. The duration of postischemic cardiac fibrillation is given in seconds. Concentrations of tissue metabolites from isolated hearts are expressed in micromoles per gram of dry weight.

Statistical analysis. All multiple comparisons in study I were performed by analysis of variance. When significant $\mathrm{F}$ values were calculated, intergroup comparisons were performed with Duncan's test. HPLC data of study II were analyzed by means of the Mann-Whitney test.

\section{Results}

\section{Study I}

Cardiac function. Table I shows the absolute values for preischemic cardiac function; no statistically signif- 
Table II. Postischemic functional recovery rates and duration of fibrillation during the initial phase of reperfusion after 2 hours of global ischemia at $10^{\circ} \mathrm{C}$

\begin{tabular}{|c|c|c|c|c|c|}
\hline Group & $\begin{array}{c}\text { Maximum LVDP } \\
(\%)\end{array}$ & $\begin{array}{c}d P / d t m a x \\
(\%)\end{array}$ & $\begin{array}{c}\text { Heart rate } \\
(\%)\end{array}$ & $\begin{array}{c}\text { Coronary flow } \\
(\%)\end{array}$ & $\begin{array}{c}\text { Fibrillation } \\
(s)\end{array}$ \\
\hline \multicolumn{6}{|l|}{ Protocol 1} \\
\hline ST alone & $71.1 \pm 9.5$ & $73.7 \pm 2.2 \dagger$ & $76.8 \pm 3.1$ & $82.0 \pm 5.1$ & $180 \pm 13$ \\
\hline $\mathrm{ST}+\mathrm{DFO}$ & $83.7 \pm 1.5$ & $74.2 \pm 3.9$ & $85.2 \pm 2.8$ & $92.2 \pm 2.6$ & $126 \pm 15^{*}$ \\
\hline $\mathrm{ST}+\mathrm{Zn}$ & $80.0 \pm 2.8$ & $76.4 \pm 3.5$ & $87.6 \pm 4.9$ & $89.9 \pm 2.7$ & $134 \pm 12 *$ \\
\hline $\mathrm{ST}+\mathrm{Zn}-\mathrm{DFO}$ & $86.4 \pm 2.1$ & $82.6 \pm 2.7$ & $90.4 \pm 1.1$ & $94.8 \pm 2.0$ & $116 \pm 14^{*}$ \\
\hline $\mathrm{ST}+\mathrm{Ga}$ & $81.2 \pm 1.9$ & $72.2 \pm 3.3$ & $88.0 \pm 2.6$ & $83.0 \pm 4.1$ & $139 \pm 9 *$ \\
\hline $\mathrm{ST}+\mathrm{Ga}-\mathrm{DFO}$ & $80.4 \pm 1.0$ & $76.3 \pm 3.2$ & $85.4 \pm 3.6$ & $86.8 \pm 3.7$ & $106 \pm 10 *$ \\
\hline \multicolumn{6}{|l|}{ Protocol 2} \\
\hline ST alone & $40.2 \pm 15.4$ & $30.3 \pm 10.7$ & $42.1 \pm 16.7$ & $47.7 \pm 18.9$ & $278 \pm 39$ \\
\hline $\mathrm{ST}+\mathrm{DFO}$ & $81.5 \pm 1.1$ & $69.2 \pm 2.3^{*}$ & $87.6 \pm 3.6$ & $89.7 \pm 2.1$ & $116 \pm 6^{*}$ \\
\hline $\mathrm{ST}+\mathrm{Zn}$ & $79.0 \pm 2.8$ & $79.2 \pm 2.2^{*}$ & $83.8 \pm 6.5$ & $85.9 \pm 2.9$ & $129 \pm 12 *$ \\
\hline $\mathrm{ST}+\mathrm{Zn}-\mathrm{DFO}$ & $89.4 \pm 1.5^{*}$ & $79.7 \pm 3.5^{*}$ & $84.3 \pm 3.9$ & $91.5 \pm 2.1$ & $113 \pm 9 *$ \\
\hline $\mathrm{ST}+\mathrm{Ga}$ & $82.5 \pm 1.6$ & $69.2 \pm 5.9^{*}$ & $92.0 \pm 1.7$ & $91.0 \pm 3.7$ & $110 \pm 8^{*}$ \\
\hline $\mathrm{ST}+\mathrm{Ga}-\mathrm{DFO}$ & $79.2 \pm 1.6$ & $70.5 \pm 2.6^{*}$ & $82.7 \pm 2.5$ & $86.3 \pm 2.7$ & $98 \pm 7 *$ \\
\hline
\end{tabular}

For legend see Table I.

$* P<.05$ versus corresponding ST-alone group.

$\dagger P<.05$ versus ST-alone group (protocol 2 ).

icant differences were found among any of the study groups. Table II and Fig 2 show the postischemic recovery of cardiac function and the duration of fibrillation from the onset of reperfusion until spontaneous conversion to sinus rhythm. Protection by intermittent administration of unsupplemented ST (ST alone, protocol 1) resulted in $29.8 \% \pm 0.5 \%$ recovery of aortic flow when compared with the preischemic value (Fig 2 ). When hearts were protected with ST alone according to protocol 2, however, aortic flow recovery was reduced $(8.0 \% \pm 4.0 \% ; P<.05)$. With protocol 1 , significant improvement was observed when ST was supplemented with DFO, zinc, and gallium. Using the combination of zinc-DFO and also gallium-DFO, aortic flow recovery rates were even superior $(P<.05)$ to those obtained by supplementation of DFO, zinc, or gallium alone. The same pattern was observed when protocol 2 was used: The highest recoveries of aortic flow were measured in hearts protected by ST supplemented with zinc-DFO or gallium-DFO. The recovery rates of the parameters maximum left ventricular developed pressure and $\mathrm{dP} / \mathrm{dtmax}$ mirrored the aortic flow data such that protection by intermittent unsupplemented ST (protocol 1) resulted in significantly improved recovery when compared with the corresponding group using protocol 2 . With the latter protocol, any of the supplements to the baseline cardioplegic solution led to significant improvement of both maximum left ventricular developed pressure and dP/dtmax. Even unsupplemented cardioplegic solution administered according to protocol 1 resulted in recovery rates of these $2 \mathrm{pa}-$ rameters as high as $71.1 \%$ and $73.7 \%$. When this pro- tocol was used, no further improvement was observed with any of the supplements tested.

Except for 1 group (ST alone, protocol 2), postischemic recovery of heart rate measured between $76.8 \%$ and $92.0 \%$. No statistically significant differences were observed among any of the experimental groups. Postischemic recoveries of coronary flow ranged between $47.7 \%$ and $94.8 \%$ of preischemic values. Group-dependent numeric differences in mean values of coronary flow recovery failed to reach statistical significance. All hearts defibrillated spontaneously within 10 minutes of Langendorff reperfusion after 2 hours of global hypothermic ischemia. With protocol 1, supplementation of the cardioplegic solution with DFO, zinc, zinc-DFO, gallium, and gallium-DFO reduced the duration of postischemic fibrillation significantly when compared with the time span measured with the use of the unsupplemented variant. The same pattern was observed with protocol 2. Each supplement to baseline cardioplegic solution resulted in a significant reduction of the postischemic fibrillation period. Administration of DFO in its complexes with zinc or gallium further reduced the mean values of the fibrillation periods when compared with the supplementation with zinc, gallium, or DFO alone. However, these numeric differences in mean values were not statistically significant.

Creatine kinase leakage. There was no significant difference in the preischemic baseline creatine kinase leakage among any of the experimental groups (Table III). When compared with preischemic values, immediate postischemic creatine kinase leakage was increased in all experimental groups. All supplements to cardio- 
Table III. Creatine kinase leakage during the preischemic and postischemic perfusion periods

\begin{tabular}{|c|c|c|c|}
\hline Group & $\begin{array}{l}\text { Preischemic, working heart } \\
\text { (IU/5 min/heart) }\end{array}$ & $\begin{array}{c}\text { Postischemic, Langendorff } \\
\text { (IU/5 min/heart) }\end{array}$ & $\begin{array}{c}\text { Postischemic, working heart } \\
\text { (IU/5 min/heart) }\end{array}$ \\
\hline \multicolumn{4}{|l|}{ Protocol 1} \\
\hline ST alone & $0.25 \pm 0.01$ & $0.93 \pm 0.08$ & $0.38 \pm 0.03$ \\
\hline $\mathrm{ST}+\mathrm{DFO}$ & $0.25 \pm 0.01$ & $0.62 \pm 0.3^{*}$ & $0.20 \pm 0.01$ \\
\hline $\mathrm{ST}+\mathrm{Zn}$ & $0.29 \pm 0.02$ & $1.10 \pm 0.13$ & $0.43 \pm 0.03$ \\
\hline $\mathrm{ST}+\mathrm{Zn}-\mathrm{DFO}$ & $0.26 \pm 0.01$ & $0.42 \pm 0.02 \dagger$ & $0.28 \pm 0.02$ \\
\hline $\mathrm{ST}+\mathrm{Ga}$ & $0.24 \pm 0.01$ & $0.63 \pm 0.04 *$ & $0.35 \pm 0.03$ \\
\hline $\mathrm{ST}+\mathrm{Ga}-\mathrm{DFO}$ & $0.23 \pm 0.01$ & $0.53 \pm 0.05^{*}$ & $0.29 \pm 0.02$ \\
\hline \multicolumn{4}{|l|}{ Protocol 2} \\
\hline ST alone & $0.24 \pm 0.01$ & $1.05 \pm 0.09$ & $0.52 \pm 0.06$ \\
\hline $\mathrm{ST}+\mathrm{DFO}$ & $0.23 \pm 0.01$ & $0.63 \pm 0.05^{*}$ & $0.24 \pm 0.02$ \\
\hline $\mathrm{ST}+\mathrm{Zn}$ & $0.27 \pm 0.02$ & $0.90 \pm 0.05$ & $0.41 \pm 0.03$ \\
\hline $\mathrm{ST}+\mathrm{Zn}-\mathrm{DFO}$ & $0.23 \pm 0.01$ & $0.43 \pm 0.02 \dagger$ & $0.25 \pm 0.01$ \\
\hline $\mathrm{ST}+\mathrm{Ga}$ & $0.23 \pm 0.04$ & $0.63 \pm 0.03^{*}$ & $0.31 \pm 0.01$ \\
\hline $\mathrm{ST}+\mathrm{Ga}-\mathrm{DFO}$ & $0.24 \pm 0.01$ & $0.52 \pm 0.06^{*}$ & $0.32 \pm 0.03$ \\
\hline
\end{tabular}

For legend, see Table I.

$* P<.05$ versus corresponding group ST alone.

$\dagger P<.05$ versus corresponding groups ST alone, $\mathrm{ST}+\mathrm{DFO}$, and $\mathrm{ST}+\mathrm{Zn}$.

plegic solution with the exception of zinc alone were found to be associated with significantly smaller increases of creatine kinase leakage during reperfusion in the Langendorff mode. This observation was independent of the protocol used. The lowest creatine kinase concentrations were measured in effluents from hearts protected by ST supplemented with zinc-DFO and gallium-DFO. Subsequent reperfusion in the working heart mode was accompanied by a marked decrease in the creatine kinase leakage in all experimental groups. During this period, no significant differences were observed among any of the study groups.

\section{Study II}

HPLC analyses. Table IV summarizes the HPLC measurements of malondialdehyde, ascorbic acid, oxypurine, and nucleoside in hearts subjected to regional or global ischemia. Group 1 comprises results from nonischemic baseline controls, whereas hearts in groups 3 and 5 were treated with gallium-DFO complex. Hearts in groups 2 and 4 served as ischemic controls perfused with unsupplemented perfusate.

Regional ischemia accomplished by temporary occlusion of the left anterior descending coronary artery and subsequent reperfusion with unsupplemented perfusate resulted in an increase in tissue malondialdehyde levels from 0.047 to $0.240 \mu \mathrm{mol} / \mathrm{g}$ dry weight $(P<.05)$. This increase was coupled with a decrease in tissue ascorbic acid levels from 1.191 to $0.079 \mu \mathrm{mol} / \mathrm{g}$ dry weight. In addition, regional ischemia went along with a marked decrease in myocardial concentrations of the energy-rich phosphates ATP, ADP, and guanosine triphosphate, whereas catabolites of the former, such as xanthine and adenosine, were increased in concentration. When the perfusate was supplemented with gallium-DFO, however, ischemia-induced changes were significantly less pronounced with regard to malondialdehyde, uric acid, inosine, ATP, ADP, and adenosine.

Similar results were obtained in hearts subjected to global ischemia: malondialdehyde levels increased after 35 minutes of normothermic ischemia, again coupled with a decrease in postischemic concentrations of ascorbate. Tissue concentrations of ATP and ADP decreased after ischemia and subsequent reperfusion. Accordingly, xanthine and adenosine were found to be markedly increased. Supplementation of the galliumDFO complex during the perfusion periods caused a tendency toward a reverse in these ischemia-associated changes. For example, the malondialdehyde levels increased from a basal tissue concentration of 0.047 to $0.892 \mu \mathrm{mol} / \mathrm{g}$ dry weight after 35 minutes of global ischemia, whereas this increase was only half as pronounced in the presence of the gallium-DFO complex.

\section{Discussion}

DFO alone and in its complexes with zinc or gallium were assessed for cardioprotective properties in isolated perfused rat hearts. The use of zinc-DFO and gallium-DFO as additives to crystalloid cardioplegic solution enhanced postischemic functional recovery in the working heart model, attenuated creatine kinase leakage, and shortened the duration of ventricular fibrillation during reperfusion after global hypothermic ischemia. Treatment with gallium-DFO proved to reduce postischemic tissue malondialdehyde levels, 
which were measured as an index of lipid peroxidation. The results suggest (1) that the use of DFO in its complex with zinc and gallium affords additional protection when compared with the effects of the single components alone and (2) that this effect is due to reduced postischemic generation of oxygen-derived intermediates.

Previous studies have implicated an important role of reactive oxygen-derived intermediates such as the superoxide anion, hydrogen peroxide, and the hydroxyl radical in myocardial injury resulting from ischemia and subsequent reperfusion. ${ }^{1}$ The conversion of superoxide anion radicals to the highly reactive hydroxyl radicals is thought to require trace amounts of redox-active transition metals such as iron and copper via the metal catalyzed Haber-Weiss reaction. ${ }^{3}$ The burst of iron and copper ions we observed previously on postischemic resumption of coronary flow supports this hypothesis and is in accord with the increase of hydroxyl radical activity in postischemic tissues. ${ }^{18} \mathrm{~A}$ specific chelator for transition metal ions such as DFO can tightly bind both ions, thereby modulating their redox potential. This pull-out mechanism explains the organ protective properties of DFO and other chelators of iron that we and others have described before. ${ }^{5,19}$ The data presented here support these findings in that supplementation of ST solution with $100 \mu \mathrm{mol} / \mathrm{L}$ DFO leads to significant improvement of postischemic myocardial function and reduction of ischemic injury in isolated working rat hearts.

Another strategy for prevention of hydroxyl radical formation is to displace traces of transition metals of their binding sites in the metal-catalyzed reaction by adding redox-inactive agents such as zinc ions or gallium ions to the system. These ions compete for the binding sites of redox-active iron ions, displace them, and divert the site of free radical production. We and others have found that zinc exerts antiarrhythmic effects, inhibits the site-specific formation of radicals catalyzed by transition metals, and improves myocardial protection of isolated rat hearts. ${ }^{8,20,21}$ Our present findings support these observations in that supplementation of zinc to the baseline cardioplegic solution improved recovery of postischemic aortic flow and shortened the duration of postischemic fibrillation when compared with the results with unsupplemented cardioplegic solution.

Even though the role of zinc in myocardial ischemia is not fully understood, the various data sets suggest that resident zinc ions disclose an endogenous protective effect against organ damage induced by oxygenderived free radicals and that there is an increased requirement for zinc in the postischemic heart. ${ }^{22}$ In human beings, plasma zinc levels were found to be reduced after acute myocardial infarction. ${ }^{23}$ In experimental models involving organ ischemia, an increased uptake of zinc into mitochondria and microsomal fractions, both possible sites of production of reactive oxygen-derived intermediates, has been observed. ${ }^{24}$

The effects of gallium, another redox-inactive ion species, on the cardiovascular and other organ systems have been studied less intensively. Gallium nitrate is effective in the treatment of hypercalcemia and advanced neoplasia. ${ }^{25,26}$ It also inhibits norepinephrine-induced contractions in rabbit aortic strips but does not affect those elicited by high potassium solution. ${ }^{27}$ Millart and coworkers ${ }^{28}$ observed that administration of $\mathrm{GaCl}_{3}$ improves cardiac function in normoxic isolated rat hearts. Interestingly, $\mathrm{GaCl}_{3}$ was shown to decrease the free radical production in neonatal rat heart cells exposed to oxidative stress. ${ }^{9}$ In accordance with our results with zinc, protection by baseline cardioplegic solution supplemented with gallium was followed by significant improvement of postischemic recovery of aortic flow and reduction of ischemic injury when compared with the use of unsupplemented cardioplegic solution. Similar biochemical properties of zinc and gallium suggest the same pathway of competitive iron and copper replacement, and our own observations support previous results indicative of the cardioprotective properties of both ion species.

Rather than assessment of zinc, gallium, and DFO alone, our purpose was to reveal whether administration of DFO in its complex with zinc and also gallium acts synergistically in terms of myocardial protection by virtue of the combined effects of the push-and-pull mechanisms. ${ }^{7}$ The proposed site of its action is interference with the transition metal-dependent generation of hydroxyl radicals. The "pull" component of the protection by zinc-DFO (and likewise gallium-DFO) is achieved by the chelation of ferric iron ions by DFO. $\mathrm{Fe}^{+3}$ has very high affinity for DFO so that $\mathrm{Fe}^{+3}$ (available) + zinc-DFO results in Fe-DFO + zinc $^{+2}$. The "push" part of the protective effect is attained through the action of the non-DFO zinc on other sites where redox-active metal ions reside. Thus, additional $\mathrm{Fe}^{+3}$, and possibly $\mathrm{Cu}^{+2}$, can be displaced from their sites by the previously "liberated" zinc ions. Because zinc is not redox-active, this is expected to further reduce hydroxyl radical formation. In addition, the combination of zinc or gallium with DFO has another potential advantage: DFO, in itself, is a relatively large and randomly oriented molecule with low ability to penetrate into cells. On metal binding, however, DFO within its 
Table IV. HPLC analyses of metabolites from isolated rat hearts after reperfusion after regional and global ischemia

\begin{tabular}{|c|c|c|c|c|c|}
\hline & \multirow{2}{*}{$\begin{array}{c}\text { No ischemia } \\
\text { Baseline } \\
\text { (group } 1, n=4 \text { ) }\end{array}$} & \multicolumn{2}{|c|}{ Regional ischemia } & \multicolumn{2}{|c|}{ Global ischemia } \\
\hline & & $\begin{array}{c}\text { Control } \\
\text { (group 2, } n=7 \text { ) }\end{array}$ & $\begin{array}{c}\text { Ga-DFO } \\
\text { (group } 3, n=5 \text { ) }\end{array}$ & $\begin{array}{c}\text { Control } \\
\text { (group } 4, n=3 \text { ) }\end{array}$ & $\begin{array}{c}G a-D F O \\
\text { (group 5, } n=4 \text { ) }\end{array}$ \\
\hline MDA & $0.047 \pm 0.006$ & $0.240 \pm 0.014 *$ & $0.104 \pm 0.027 \dagger$ & $0.892 \pm 0.010$ & $0.497 \pm 0.088$ \\
\hline Ascorbic acid & $1.191 \pm 0.489$ & $0.079 \pm 0.020 *$ & $0.124 \pm 0.036$ & $0.562 \pm 0.035$ & $1.532 \pm 0.169$ \\
\hline ATP & $27.69 \pm 1.20$ & $7.150 \pm 0.590 *$ & $13.15 \pm 0.970 \dagger$ & $3.913 \pm 0.499$ & $4.889 \pm 0.710$ \\
\hline ADP & $8.760 \pm 0.130$ & $4.030 \pm 0.200^{*}$ & $5.660 \pm 0.450 \dagger$ & $3.694 \pm 0.192$ & $2.749 \pm 0.148$ \\
\hline AMP & $0.566 \pm 0.021$ & $0.670 \pm 0.070$ & $0.900 \pm 0.080$ & $2.095 \pm 0.295$ & $0.518 \pm 0.114$ \\
\hline Adenosine & $0.110 \pm 0.015$ & $1.025 \pm 0.106^{*}$ & $0.669 \pm 0.114 \dagger$ & $0.683 \pm 0.083$ & $0.395 \pm 0.017$ \\
\hline$\sum \mathrm{A}$ & $37.02 \pm 1.350$ & $11.85 \pm 0.860 *$ & $19.71 \pm 1.500 \dagger$ & $9.697 \pm 0.986$ & $8.156 \pm 0.971$ \\
\hline Energy charge & $0.870 \pm 0.003$ & $0.770 \pm 0.060$ & $0.810 \pm 0.060$ & $0.590 \pm 0.060$ & $0.770 \pm 0.100$ \\
\hline Inosine & $0.037 \pm 0.012$ & $0.775 \pm 0.098^{*}$ & $0.397 \pm 0.022 \dagger$ & $6.540 \pm 1.553$ & $5.465 \pm 0.606$ \\
\hline Hypoxanthine & $0.043 \pm 0.005$ & $0.385 \pm 0.072 *$ & $0.171 \pm 0.068$ & $0.300 \pm 0.015$ & $0.131 \pm 0.023$ \\
\hline Xanthine & $0.023 \pm 0.001$ & $0.373 \pm 0.067 *$ & $0.231 \pm 0.062$ & $1.340 \pm 0.107$ & $0.986 \pm 0.070$ \\
\hline Uric acid & $0.014 \pm 0.004$ & $0.132 \pm 0.024 *$ & $0.056 \pm 0.010 \dagger$ & $0.323 \pm 0.007$ & $0.152 \pm 0.024$ \\
\hline
\end{tabular}

complexes with zinc or gallium assumes a well-defined globular structure. ${ }^{29}$ This alteration is expected to render these complexes more permeable into cells than the metal-free species. ${ }^{7}$ In noncardiac test systems involving free radical-induced injury triggered by radiation or alkali burn, we were able to prove the protective efficacy of the push-and-pull mechanism using both zincDFO and gallium-DFO with regard to hydroxyl radical-induced tissue damage. ${ }^{10-13,30}$ In compliance with these results, use of zinc-DFO enhanced functional recovery of isolated rat hearts after normothermic ischemia. ${ }^{31}$ To date, no study has been done on the effects of DFO in its complexes with zinc or gallium involving hypothermic myocardial ischemia, such as hearts frequently undergo during routine cardiac surgery. Thus, we used 2 protocols of hypothermic cardiac arrest. Protocol 1 represented an in vitro model of intermittent cardioplegic perfusion during hypothermic ischemia, whereas protocol 2 served as a model of hypothermic arrest with initial induction by administration of cardioplegic solution and no further interventions except for a single-shot washout directly before reperfusion. Intermittent application of unsupplemented ST afforded superior recovery of functional parameters as compared with recovery rates obtained after its application according to protocol 2 . This result is compatible with the findings of others that multidose regimens using this solution during ischemic intervals up to 2 hours lead to drastic improvement of functional and metabolic recovery when compared with single-dose applications. It was suggested that the reinfusions help to wash out acid metabolites that inhibit anaerobic metabolism, replace substrate, and maintain an ideal $\mathrm{pH}$ for continued metabolism at a low temperature. ${ }^{32} \mathrm{In}$ accordance with the data obtained with unsupplemented ST, the numeric mean values of the functional recovery rates tended to be higher with experimental protocol 1, regardless of the modifications of the baseline cardioplegic solution. This result is also indicative of the superiority of the multidose regimen.

The recovery rates of the parameter aortic flow were constantly lower than those of other parameters (maximum left ventricular developed pressure and $\mathrm{dP} / \mathrm{dtmax}$ ). Compared with the latter, however, the regeneration of aortic flow appears more reliable because hearts can generate a considerable rate of recovery in developed pressure, which still may not be high enough to overcome the afterload imposed in a working heart system. This connection also explains the dissociation of the relatively high recovery rates in maximum left ventricular developed pressure and dP/dtmax from lower recoveries in aortic flow.

Study I clearly demonstrates that both the zinc-DFO complex and the gallium-DFO complex significantly improve functional recovery after 2 hours of global hypothermic ischemia when compared with the protective effects of either constituent alone. Protection enhancement is very likely to result from a combination of push-and-pull mechanisms, thereby preventing site-specific metal-mediated oxidative tissue damage. 
This thesis is strongly supported by measurements of reduced malondialdehyde levels in hearts of study II treated with gallium-DFO. Malondialdehydes originate from an oxygen-derived free radical-mediated breakdown of phospholipids and represent a possible quantifiable marker of molecular damage occurring on reperfusion. Not surprisingly, concentration changes in malondialdehydes were found to be coupled to an increase in the antioxidative capacity as measured by tissue ascorbate levels (Table IV). Above that, as response to oxygen and substrate deprivation, normothermic ischemia leads to the typical concentration changes in energy-rich phosphates and their dephosphorylated catabolites such as oxypurines and nucleotides.

The concentrations of DFO in complex with zinc or gallium selected in studies I and II were low (5 $\mu \mathrm{mol} / \mathrm{L})$ when compared with those for treatment with the single components (DFO, $50 \mu \mathrm{mol} / \mathrm{L}$; zinchistidinate, $30 \mu \mathrm{mol} / \mathrm{L}$; gallium-nitrate, $100 \mu \mathrm{mol} / \mathrm{L})$. Concentrations were selected in view of previous evidence that zinc-DFO and gallium-DFO are effective in a range between 5 and $30 \mu \mathrm{mol} / \mathrm{L}$ whereas zinc alone and DFO alone are effective only in much higher concentrations. $8,10,33$

\section{Limitations of the study}

There are many obvious differences between the isolated perfused rat heart and the human heart in situ. The limitations of this type of perfusion system include the lack of a physiologic peripheral component and aspects of metabolic, nervous, and hormonal control because aqueous solutions free of red cells, protein, and fatty acids are used to perfuse the coronary arteries. In addition, the time of reperfusion is limited as is the time for postischemic observations.

It was our purpose to focus independently on functional effects in the one study and on changes in metabolism in the other. The fact that studies I and II were conducted in laboratories using different equipment, protocols, and rat strains, however, may limit the comparability of results even though appropriate controls have been used in each set of experiments.

\section{Conclusions}

On the basis of the considerable impact of transition metals on the generation of oxygen-derived free radical formation, we have observed that the iron chelator DFO and nonredox active ions such as zinc and gallium enhance postischemic myocardial function when added to baseline cardioplegic solution. The results obtained with DFO in its complexes with zinc or galli- um were even superior to the recovery rates achieved with either of the components alone. Previous evidence from studies on the protective effects of DFO in its complexes with zinc or gallium and our measurements of metabolic changes representing sequelae of oxidative tissue damage suggest an antioxidative synergism of the push-and-pull mechanism to be involved in the current findings. ${ }^{10-13,33}$ Whether this new approach to myocardial protection will find clinical usefulness during postischemic reperfusion of the heart or in cardioplegic solutions remains to be determined.

We express our gratitude to Dr N. Kitrossky for his help in the HPLC analyses and to C. Abraham, PhD, our consultant statistician for biostatistical review of the study.

Received for publication June 21, 2000; revisions requested Sept 21, 2000; revisions received Oct 27, 2000; accepted for publication Nov 30, 2000.

Address for reprints: Matthias Karck, MD, Department of Thoracic and Cardiovascular Surgery, Hannover Medical School, Carl Neuberg Str 1, 30623 Hannover, Federal Republic of Germany.

\section{REFERENCES}

1. Kloner RA, Przyklenk K, Wittaker P. Deleterious effects of oxygen radicals in ischemia/reperfusion: resolved and unresolved issues. Circulation 1991;80:1115-27.

2. Bagchi M, Prasad MR, Engelman RM, Das DK. Effects of free radicals on the fluidity of myocardial membranes. Free Radic Res Commun 1989;7:375-80.

3. Haber F, Weiss J. The catalytic decomposition of hydrogen peroxide by iron salts: Proc R Soc Lond 1934;A147:332-51.

4. Braughler JM, Duncan LA, Chase RL. The involvement of iron in lipid peroxidation. J Biol Chem 1986;261:10282-9.

5. Bernard M, Menasché P, Pietri S, Grousset C, Piwnica A, Cozzone PJ. Cardioplegic arrest superimposed on evolving myocardial ischemia: improved recovery after inhibition of hydroxyl radical generation by peroxidase or deferoxamine, a 31P nuclear resonance study. Circulation 1988;78(Suppl):III164-72.

6. Drossos G, Lazou A, Panagopoulos P, Westaby S. Deferoxamine cardioplegia reduces superoxide radical production in human myocardium. Ann Thorac Surg 1995;59:169-72.

7. Chevion M. Protection against free radical-induced and transition metal-mediated damage: the use of "pull" and "push" mechanism. Free Radic Res Commun 1991;12-13:691-6.

8. Powell SR, Aiuto L, Hall D, Tortolani AJ. Zinc supplementation enhances the effectiveness of St. Thomas' Hospital No. 2 cardioplegic solution in an in vitro model of hypothermic cardiac arrest. J Thorac Cardiovasc Surg 1995;110:1642-8.

9. Leperre A, Millart H, Prevost A, Kantelip JP, Lamiable D, Collery P. Gallium chloride effects on neonatal rat heart cells in culture, in standard and oxidative stress. Fund Clin Pharmacol 1994;8:563-9.

10. Banin E, Berenshtein E, Kitrossky N, Pe'er J, Chevion M. Gallium-desferrioxamine protects the cat retina against injury 
following ischemia and reperfusion. Free Radic Biol Med 2000;28:315-23.

11. Siganos CS, Frucht-Pery J, Muallem M, Berenshtein E, Naoumidi I, Ever-Hadani P, et al. Topical use of zinc desferrioxamine for corneal alkali injury in a rabbit model. Cornea 1998;17:191-5.

12. Nagler R, Marmary Y, Golan E, Chevion M. Novel protection strategy against $\mathrm{X}$-ray-induced damage to salivary glands. Radiat Res 1998;149:271-6.

13. Samuni Y, Coffin D, DeLuca A, DeGraff W, Venson D, Chevion $\mathrm{M}$, et al. The use of zinc-desferrioxamine for radioprotection in mice, tissue culture, and isolated DNA. Cancer Res 1999;59:405-

14. Neely JR, Liebermeister H, Battersby EJ, Moegen HE. Effect of pressure development on oxygen consumption by isolated rat hearts. Am J Physiol 1967;212:804-14.

15. Bergmeyer HU, editor. Methods in enzymatic analysis. London: Academic Press; 1974. p. 1777.

16. Humphrey SM, Cartner LA, Hollis DG. Critical early metabolic changes associated with myocardial recovery or failure after total ischemia in the rat heart. Basic Res Cardiol 1987;2:304-16.

17. Tavazzi B, Lazzarino G, Di Pierro D, Giardina B. Malondialdehyde production and ascorbate decrease are associated to the reperfusion of the isolated postischemic rat heart. Free Radic Biol Med 1992;13:75-8.

18. Chevion M, Jiang Y, Har-El R, Berenshtein E, Kitrossky N. Copper and iron are mobilized following myocardial ischemia: possible predictive criteria for tissue injury. Proc Natl Acad Sci U S A 1993;90:1102-6.

19. Karck M, Appelbaum Y, Schwalb H, Haverich A, Chevion M, Uretzky G. TPEN, a transition metal chelator, improves myocardial protection during prolonged ischemia. J Heart Lung Transplant 1992;11:979-85.

20. Powell SR, Saltman P, Uretzky G, Chevion M. The effect of zinc on reperfusion arrhythmias in the isolated perfused rat heart. Free Radic Biol Med 1990;8:33-46.

21. Chevion M. A site-specific mechanism for free radical induced biological damage: the essential role of redox active transition metals. Free Radic Biol Med 1988;3:27-37.

22. Bray TM, Bettger WJ. The physiologic role of zinc as an antioxidant. Free Radic Biol Med 1990;8:281-91.

23. Handjani AM, Cecil-Smith JJ, Hermann JB, Halstead JA. Serum zinc concentrations in acute myocardial infarction. Chest 1974;65:185-7.

24. Fiskum G. Mitochondrial damage during cerebral ischemia. Ann Emerg Med 1985;14:810-5.

25. Warrell RP Jr. Clinical trials of gallium nitrate in patients with cancer related hypercalcemia. Semin Oncol 1991;18:26-31.

26. Foster BJ, Clagett-Carr K, Leyland-Jones B. Gallium nitrate: the second metal with clinical activity. Cancer Treat Rep 1988;70: 1311-9.

27. Shetty SS, Weiss GS. Specific actions of gallium on norepinephrine-induced tension and associated $45 \mathrm{Ca}$ movements in rabbit aortic smooth muscle. J Pharmacol Exp Ther 1987;243:614-7.

28. Millart H, Leperre A, Kantelip JP, Collery P, Descous I, Tenqui T, et al. Acute effects of gallium chloride on ventricular function and metabolism of the Langendorff perfused rat heart. Anticancer Res 1993;13:2251-6.

29. Keberle $\mathrm{H}$. The biochemistry of desferrioxamine and its relation to iron metabolism. Ann NY Acad Sci 1964;119:758-69.

30. Ophir A, Berenshtein E, Kitrossky N, Averbukh E. Protection of the transiently ischemic cat retina by zinc-desferrioxamine. Invest Ophthalmol Vis Sci 1994;35:1212-22.

31. Yandong J, Schwalb H, Uretzky G, Chevion M. The combination of zinc and desferrioxamine provides protection of the heart via both "push" and "pull" mechanisms. J Mol Cell Cardiol 1991;23(Suppl V):S26.

32. Follette D, Fey K, Mulder D, Maloney JV, Buckberg GD. Prolonged safe aortic clamping by combining membrane stabilization, multidose cardioplegia, and appropriate $\mathrm{pH}$ reperfusion. J Thorac Cardiovasc Surg 1977;74:682-94.

33. Chevion M, Chuang L, Golenser J. Effects of zinc-desferrioxamine on Plasmodium falciparum in culture. Antimicrob Agents Chemother 1995;39:1902-5. 